\title{
NOTE ON THE PREVALENCE OF ANOPHELES.
}

\author{
By GEORGE H. F. NUTTALL, F.R.S.
}

IN a previous paper ${ }^{1}$ a record of the distribution of Anopheles in Great Britain was given, the localities mentioned including Cambridge and the surrounding country. Since 1901, Anopheles imagines have been searched for in cellars and houses during the winter, in places where they were known to congregate. During the summer a yearly search for larvae was made in various collections of water in the vicinity of Cambridge with a view to securing material for purposes of study and instruction. It appears worthy of note that there has been a notable decrease in the number of inagines caught in cellars, etc. in the winter months. The insects were fairly numerous in my own house during 1901-1902, as many as 60-100 being found hibernating in the cellar. In 1903-1904 there were fewer, only about 6 being caught. Not a single imagine has been caught in the house since that winter. Whereas Anopheles were frequently caught in houses during 1901-1903 in the warmer months, very few were encountered in 1904, and I have not found a single specimen this year. The common Anopheles in this region is A. maculipennis, as I have already recorded, A. bifurcatus being less frequently encountered. Apparently corresponding results have to be recorded with regard to Anopheles' larvae. These were certainly very numerous during the period 1900-1903, and they have become scarcer since, being apparently quite absent in certain waters where they formerly abounded. That the Anopheles are not extinguished is certain, for I have found a few larvae (A. maculipennis) in the river Cam this summer, and my friend Mr Pearce has brought me an imago from Grantchester (close to Cambridge), in addition to larvae of A. bifurcatus which must have hibernated there. Hibernating larvae of this species have twice been found by me since I first recorded their presence about Cambridge.

1 "Studies in Relation to Malaria," this Journal, vol. I, pp. 4-44, 1901. 
486

Note on the prevalence of Anopheles

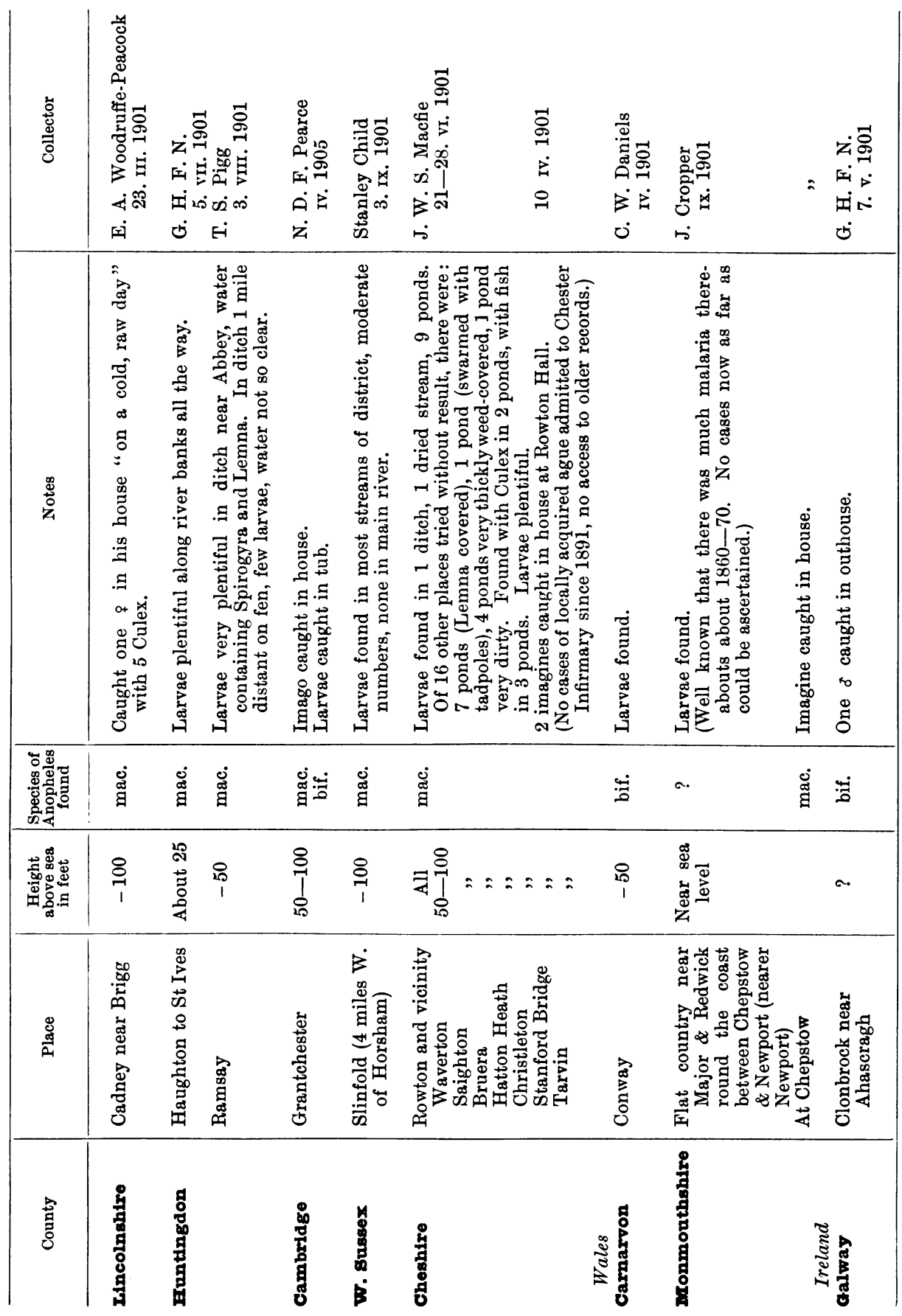


Of course this is but an isolated observation, and necessarily it can have but limited value. It is known of other insects that they are more numerous in some years than in others. For instance Culex pipiens abounded in many parts of Cambridge last year, so much so that many persons spoke to me of it, and I captured large numbers in cellars where Anopheles had previously been frequent. In one cellar, in the winter of 1903-1904, I only succeeded in catching one A. maculipennis, whilst C. pipiens were present in hundreds. We do not know the laws that govern these variations in the number of insects in different years, but it is obvious that variations do occur. It is possible that the reduction in the number of Anopheles in these parts has been due to repeated sudden rainfalls during the last three summers, personally I am strongly inclined to the opinion that these rainfalls have exerted an influence.

Sudden rainfalls would necessarily wash away the larvae in large numbers from the situations which they normally inhabit. It is dangerous to argue from an isolated instance, but knowing that ague formerly prevailed in these parts an explanation of its disappearance may possibly be found in a reduction of the Anopheles persisting for a sufficient length of time to break the chain of parasitism of the malarial parasites in man and the mosquito. It appears again warranted to suggest the desirability of further observations being made along these lines elsewhere.

The preceding data may be added to those already published regarding the geographical distribution of Anopheles in Great Britain ${ }^{1}$, the same form of tabulation being adopted here.

I am much indebted to the gentlemen mentioned in the foregoing table for kindly supplying me with the data therein contained. I determined all the specimens collected with the exception of the larvae found by Drs Daniels and Cropper, and the imago of A. maculipennis caught by the latter. Both of these gentlemen are well-known as experienced collectors of mosquitoes. I shall be indebted to any gentlemen who will kindly supply me with further facts regarding the geographical distribution of Anopheles in Great Britain. 